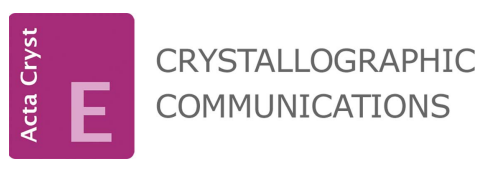

ISSN 2056-9890
Received 8 October 2021

Accepted 18 October 2021

Edited by L. Van Meervelt, Katholieke Universiteit Leuven, Belgium

Keywords: crystal structure; short inter $\mathrm{HL} \cdots \mathrm{HL}$ contact; $\mathrm{C}-\mathrm{Cl} \cdots \pi$ interactions; face-to-face $\pi$ $\pi$ stacking interactions; Hirshfeld surface analysis.

CCDC reference: 2116300

Supporting information: this article has supporting information at journals.iucr.org/e

\section{Crystal structure and Hirshfeld surface analysis of (E)-1-[2,2-dichloro-1-(4-fluorophenyl)ethenyl]-2- (2,4-dichlorophenyl)diazene}

\author{
Namiq Q. Shikhaliyev, ${ }^{\text {a }}$ Kadiriye Özkaraca, ${ }^{\mathrm{b}}$ Mehmet Akkurt, ${ }^{\mathrm{c}}$ Xanim N. Bagirova, ${ }^{\mathrm{a}}$ \\ Gulnar T. Suleymanova, ${ }^{a}$ Mirjalil S. Abdulov ${ }^{a}$ and Sixberth Mlowe ${ }^{\mathrm{d} *}$
}

\author{
aOrganic Chemistry Department, Baku State University, Z. Khalilov str. 23, AZ 1148 Baku, Azerbaijan, b Institute of \\ Natural and Applied Science, Erciyes University, 38039 Kayseri, Turkey, 'Department of Physics, Faculty of Sciences, \\ Erciyes University, 38039 Kayseri, Turkey, and ${ }^{\mathbf{d}}$ University of Dar es Salaam, Dar es Salaam University College of \\ Education, Department of Chemistry, PO Box 2329, Dar es Salaam, Tanzania. *Correspondence e-mail: \\ sixberth.mlowe@duce.ac.tz
}

In the title compound, $\mathrm{C}_{14} \mathrm{H}_{7} \mathrm{Cl}_{4} \mathrm{FN}_{2}$, the dihedral angle between the 4-fluorophenyl ring and the 2,4-dichlorophenyl ring is 46.03 (19) ${ }^{\circ}$. In the crystal, the molecules are linked by $\mathrm{C}-\mathrm{H} \cdots \mathrm{N}$ interactions along the $a$-axis direction, forming a $C(6)$ chain. The molecules are further connected by $\mathrm{C}-\mathrm{Cl} \cdots \pi$ interactions and face-to-face $\pi-\pi$ stacking interactions, forming ribbons along the $a$-axis direction. Hirshfeld surface analysis indicates that the greatest contributions to the crystal packing are from $\mathrm{Cl} \cdots \mathrm{H} / \mathrm{H} \cdots \mathrm{Cl}(35.1 \%), \mathrm{H} \cdots \mathrm{H}$ $(10.6 \%), \mathrm{C} \cdots \mathrm{C}(9.7 \%), \mathrm{Cl} \cdots \mathrm{Cl}(9.4 \%)$ and $\mathrm{C} \cdots \mathrm{H} / \mathrm{H} \cdots \mathrm{C}(9.2 \%)$ interactions.

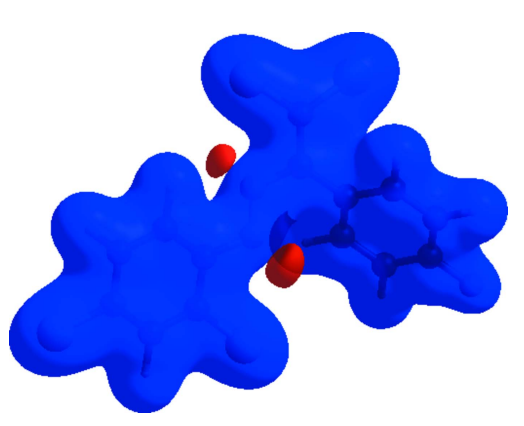

OPEN $\odot$ ACCESS

\section{Chemical context}

Azo dyes find numerous applications in a diversity of areas, including as antimicrobial agents, in molecular recognition, optical data storage, molecular switches, non-linear optics, liquid crystals, dye-sensitized solar cells, color-changing materials, etc., mainly due to the possibility of the cis-to-trans isomerization and the chromophoric properties of the $-\mathrm{N}=\mathrm{N}-$ synthon (Maharramov et al., 2018; Viswanathan et al., 2019). Not only azo-hydrazone tautomerisim, but also $E / Z$ isomerization are important phenomena in the synthetic chemistry of azo dyes (Ma et al., 2017a,b; Mahmoudi et al., 2018a,b). The design of azo dyes with functional groups led to multifunctional ligands, the corresponding transition-metal complexes of which have been used effectively as catalysts in C-C coupling and oxidation reactions (Ma et al., 2020, 2021; Mahmudov et al., 2013; Mizar et al., 2012). Moreover, the functional properties of azo dyes can be improved by attaching substituents with non-covalent bond donor or acceptor site(s) to the $-\mathrm{N}=\mathrm{N}$ - synthon (Gurbanov et al., 2020a,b; Kopylovich et al., 2011; Mahmudov et al., 2020; Shixaliyev et al., 2014). Thus, we have attached halogen-bond donor centres to the $-\mathrm{N}=\mathrm{N}-$ moiety, leading to a new azo dye, (E)-1-[2,2-dichloro-1-(4-fluorophenyl)ethenyl]-2-(2,4-dichlorophenyl)diazene, which provides multiple intermolecular non-covalent interactions.

\section{Structural commentary}

In the title compound, (Fig. 1), the dihedral angle between the 4-fluorophenyl ring $\mathrm{C} 3-\mathrm{C} 8$ and the 2,4-dichlorophenyl ring 
C9-C14 is $46.0(2)^{\circ}$. The $\mathrm{N} 2 / \mathrm{N} 1 / \mathrm{C} 1 / \mathrm{C} 2 / \mathrm{Cl} 1 / \mathrm{Cl} 2$ moiety is approximately planar, with a maximum deviation of 0.029 (1) $\AA$ for $\mathrm{Cl} 1$, and makes dihedral angles of 50.53 (18) and $11.75(18)^{\circ}$ with the $\mathrm{C} 3-\mathrm{C} 8$ and $\mathrm{C} 9-\mathrm{C} 14$ rings, respectively. In the molecule, the aromatic ring and olefin synthon adopt a trans-configuration with respect to the $\mathrm{N}=\mathrm{N}$ double bond and are almost coplanar with a $\mathrm{C} 1-\mathrm{N} 1=\mathrm{N} 2-\mathrm{C} 9$ torsion angle of $179.1(4)^{\circ}$.<smiles>Fc1ccc(C(/N=N/c2ccc(Cl)cc2Cl)=C(Cl)Cl)cc1</smiles>

\section{Supramolecular features}

In the crystal, the molecules are linked by $\mathrm{C}-\mathrm{H} \cdots \mathrm{N}$ interactions along the $a$-axis direction, forming a $C(6)$ chain (Table 1; Fig. 2; Bernstein et al., 1995). Furthermore, molecules are connected by $\mathrm{C}-\mathrm{Cl} \cdots \mathrm{Cg} 2$ interactions (Table 1 ) and faceto-face $\pi-\pi$ stacking interactions $\left[C g 1 \cdots C g 1^{\mathrm{i}}=3.873\right.$ (3) $\AA$, slippage $=1.831 \AA ⿻ C g 2 \cdots C g 2^{\mathrm{i}}=3.872(3) \AA$, slippage $=$ $1.554 \AA$; symmetry codes: (i) $x-1, y, z ;$; (ii) $x+1, y, z$; where $C g 1$ and $C g 2$ are the centroids of the 4-fluorophenyl (C3-C8) and 2,4-dichlorophenyl ring (C9-C14) rings, respectively], forming ribbons along the $a$-axis direction (Figs. 2, 3 and 4).

\section{Hirshfeld surface analysis}

Crystal Explorer (Turner et al., 2017) was used to perform a Hirshfeld surface analysis and generate the associated twodimensional fingerprint plots, with a standard resolution of the three-dimensional $d_{\text {norm }}$ surfaces plotted over a fixed colour scale of -0.1450 (red) to 1.1580 (blue) a.u (Fig. 5). In the Hirshfeld surface mapped over $d_{\text {norm }}$ (Fig. 5), the bright-red spots near atoms $\mathrm{Cl} 1, \mathrm{Cl} 3, \mathrm{H} 4, \mathrm{~N} 2$ and $\mathrm{F} 1$ indicate the short $\mathrm{C}-\mathrm{H} \cdots \mathrm{N}, \mathrm{C}-\mathrm{H} \cdots \mathrm{Cl}$ and $\mathrm{Cl} \cdots \mathrm{F}$ contacts (Table 2). Other

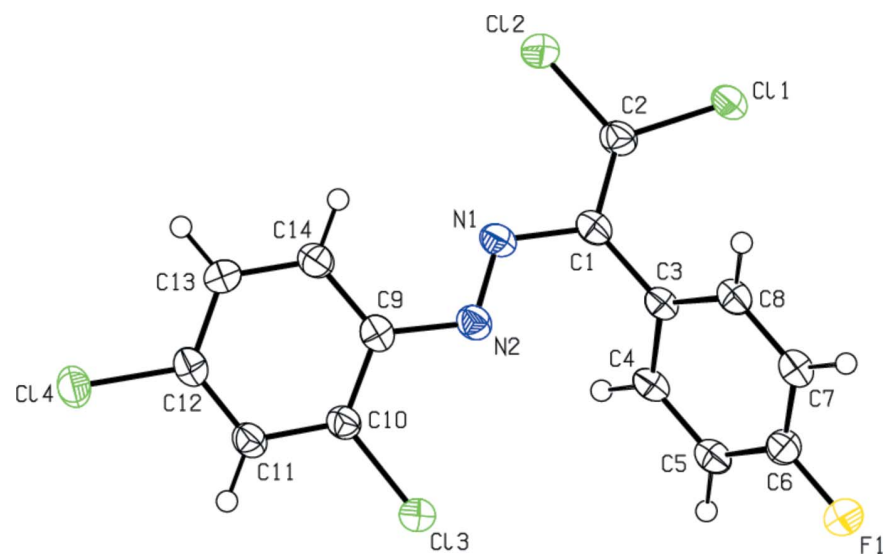

Figure 1

The molecular structure of the title compound, showing the atom labelling and displacement ellipsoids drawn at the $50 \%$ probability level.
Table 1

Hydrogen-bond geometry $\left(\AA,^{\circ}\right)$.

$C g 2$ is the centroid of the 2,4-dichlorophenyl ring (C9-C14).

\begin{tabular}{lllll}
\hline$D-\mathrm{H} \cdots A$ & $D-\mathrm{H}$ & $\mathrm{H} \cdots A$ & $D \cdots A$ & $D-\mathrm{H} \cdots A$ \\
\hline $\mathrm{C} 4-\mathrm{H} 4 \cdots \mathrm{N} 2^{\mathrm{i}}$ & 0.95 & 2.53 & $3.265(5)$ & 134 \\
$\mathrm{C} 12-\mathrm{Cl} 4 \cdots C g 2^{\mathrm{ii}}$ & $1.735(5)$ & $3.920(3)$ & $3.569(6)$ & $66.51(18)$ \\
\hline
\end{tabular}

Symmetry codes: (i) $x-1, y, z$; (ii) $x+1, y, z$.

contacts are equal to or longer than the sum of van der Waals radii. The Hirshfeld surface of the title compound mapped over the electrostatic potential (Spackman et al., 2008) is

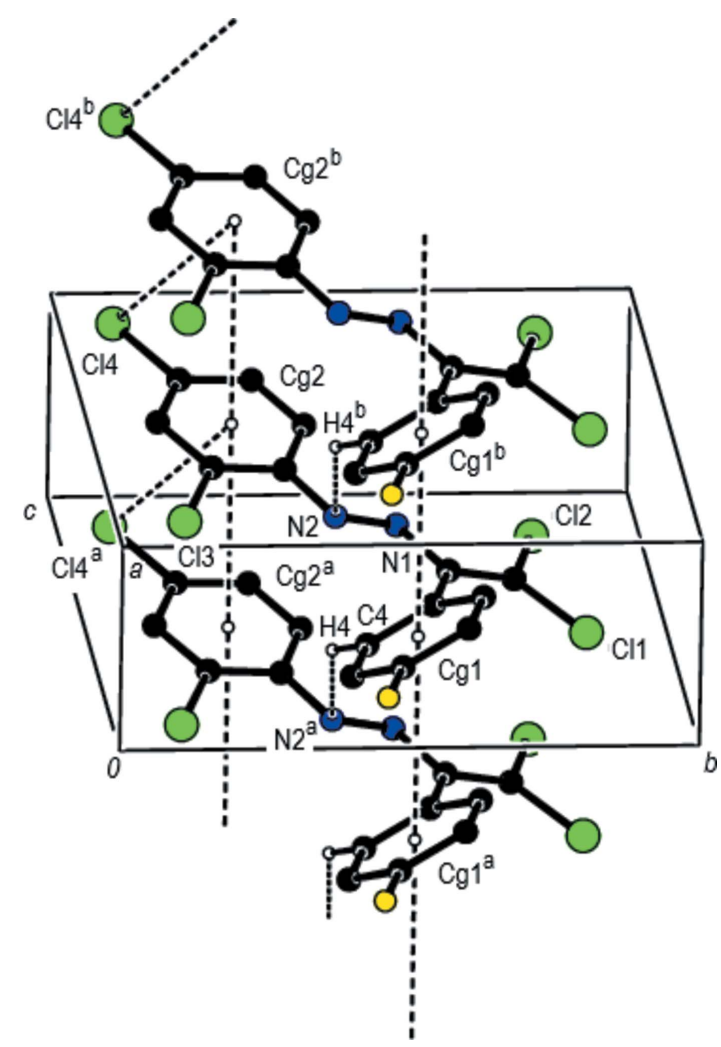

Figure 2

A general view of the intermolecular $\mathrm{C}-\mathrm{H} \cdots \mathrm{N}$ and $\mathrm{C}-\mathrm{Cl} \cdots \pi$ interactions and $\pi-\pi$ stacking interactions, shown as dashed lines. Symmetry codes: $(a)-1+x, y, z ;(b) 1+x, y, z$.

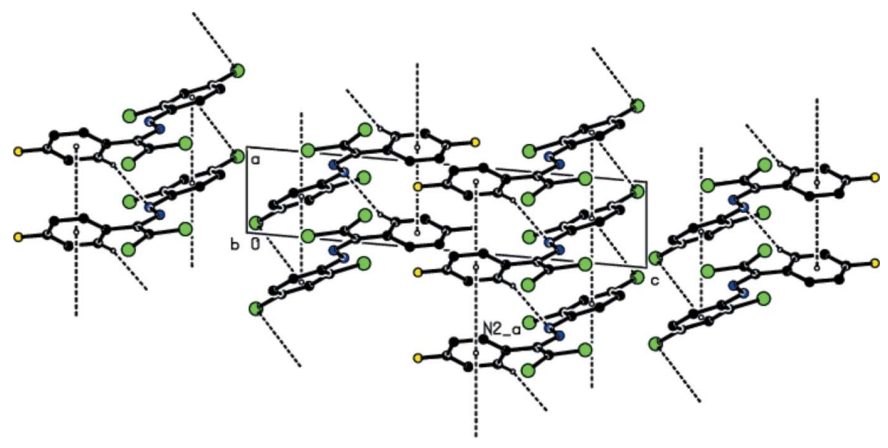

Figure 3

The crystal packing of the title compound viewed along the $b$ axis with intermolecular $\mathrm{C}-\mathrm{H} \cdots \mathrm{N}$ and $\mathrm{C}-\mathrm{Cl} \cdots \pi$ interactions and $\pi-\pi$ stacking interactions shown as dashed lines. 
Table 2

Summary of short interatomic contacts $(\AA)$ in the title compound.

\begin{tabular}{lll}
\hline Contact & Distance & Symmetry operation \\
\hline Cl1 $\cdots \mathrm{H} 11$ & 3.06 & $-1+x, 1+y, z$ \\
$\mathrm{H} 4 \cdots \mathrm{N} 2$ & 2.53 & $-1+x, y, z$ \\
$\mathrm{Cl} 1 \cdots \mathrm{F} 1$ & $3.016(3)$ & $-1-x, \frac{1}{2}+y, 1-z$ \\
$\mathrm{H} 5 \cdots \mathrm{H} 7$ & 2.55 & $-x,-\frac{1}{2}+y, 1-z$ \\
$\mathrm{Cl} 4 \cdots \mathrm{H} 13$ & 2.95 & $2-x,-\frac{1}{2}+y, 2-z$ \\
$\mathrm{Cl} 4 \cdots \mathrm{H} 14$ & 2.93 & $1-x,-\frac{1}{2}+y, 2-z$ \\
$\mathrm{Cl} 3 \cdots \mathrm{F} 1$ & $3.116(3)$ & $-x,-\frac{1}{2}+y, 1-z$ \\
\hline
\end{tabular}

shown in Fig. 6. The positive electrostatic potential (blue regions) over the surface indicates hydrogen-donor potential, whereas the hydrogen-bond acceptors are represented by negative electrostatic potential (red regions).

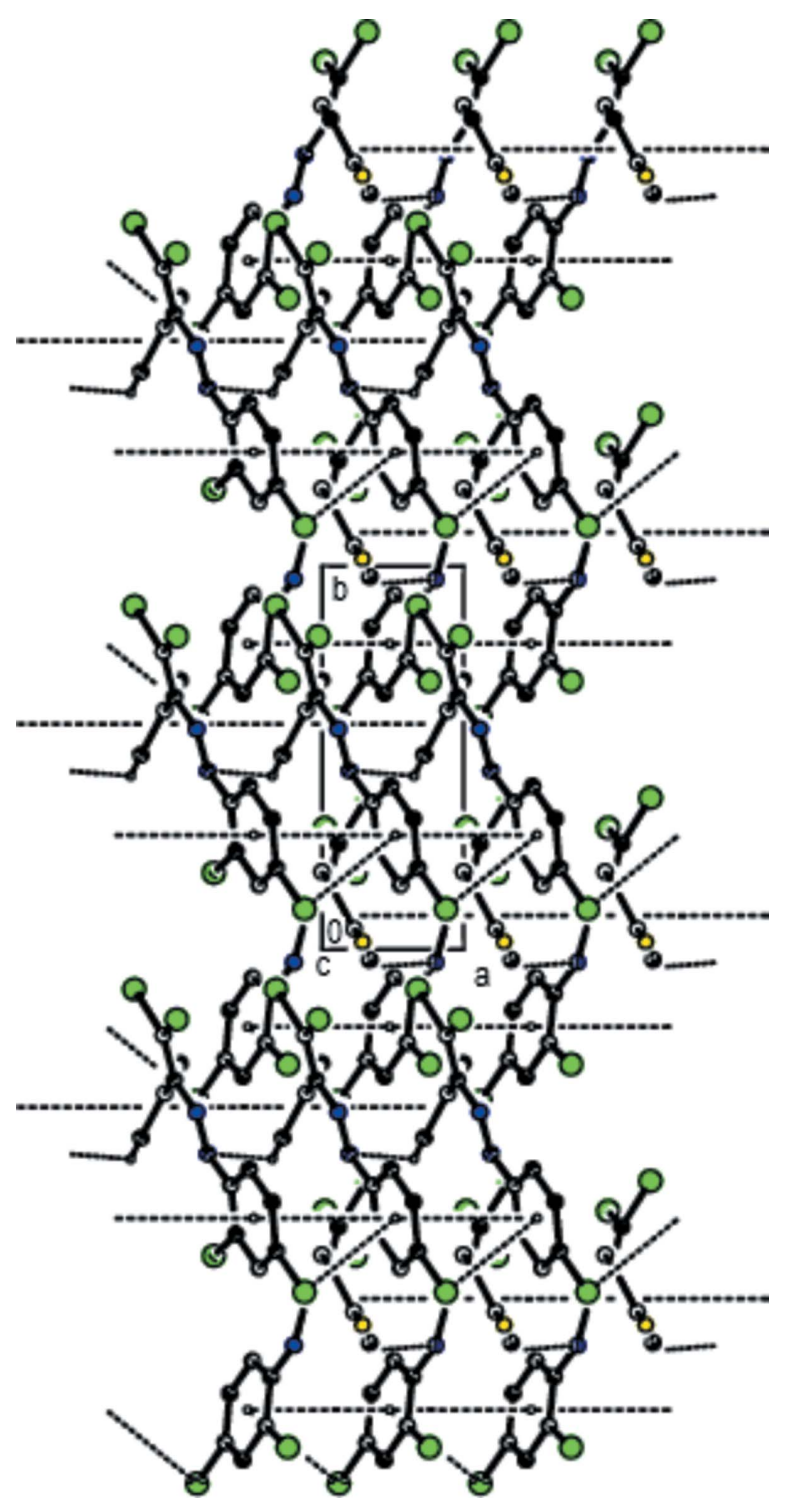

Figure 4

The crystal packing of the title compound viewed along the $c$ axis with intermolecular $\mathrm{C}-\mathrm{H} \cdots \mathrm{N}$ and $\mathrm{C}-\mathrm{Cl} \cdots \pi$ interactions and $\pi-\pi$ stacking interactions shown as dashed lines. (a)

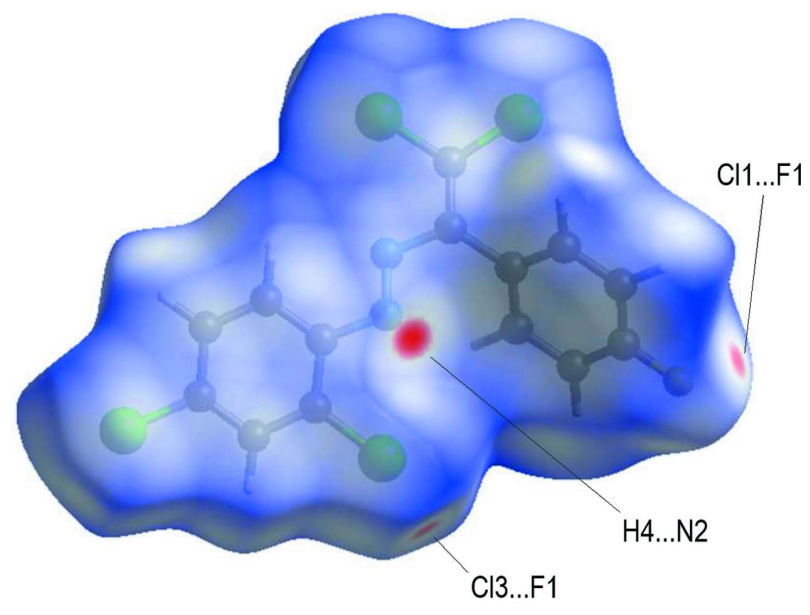

(b)

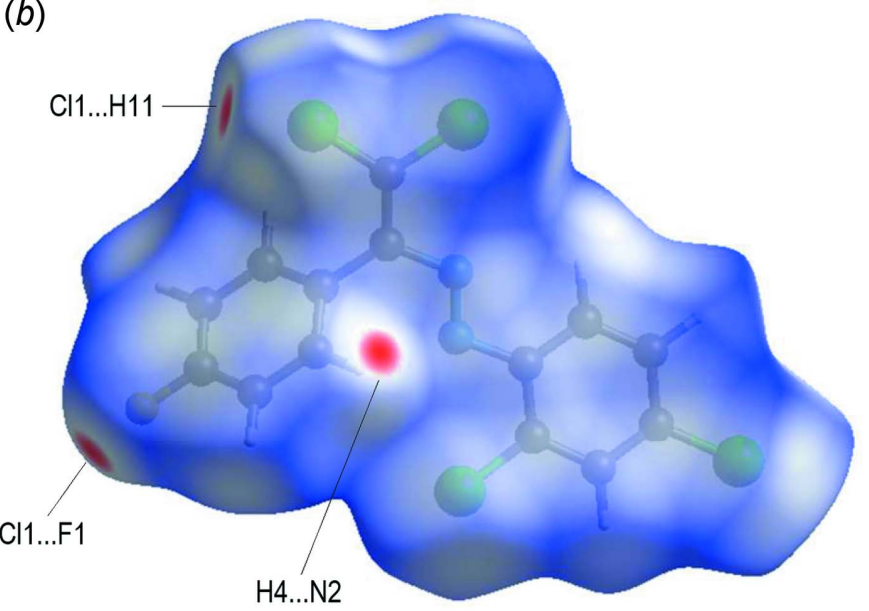

Figure 5

(a) Front and (b) back sides of the three-dimensional Hirshfeld surface of the title compound plotted over $d_{\text {norm }}$ in the range -0.1450 to 1.1580 a.u.

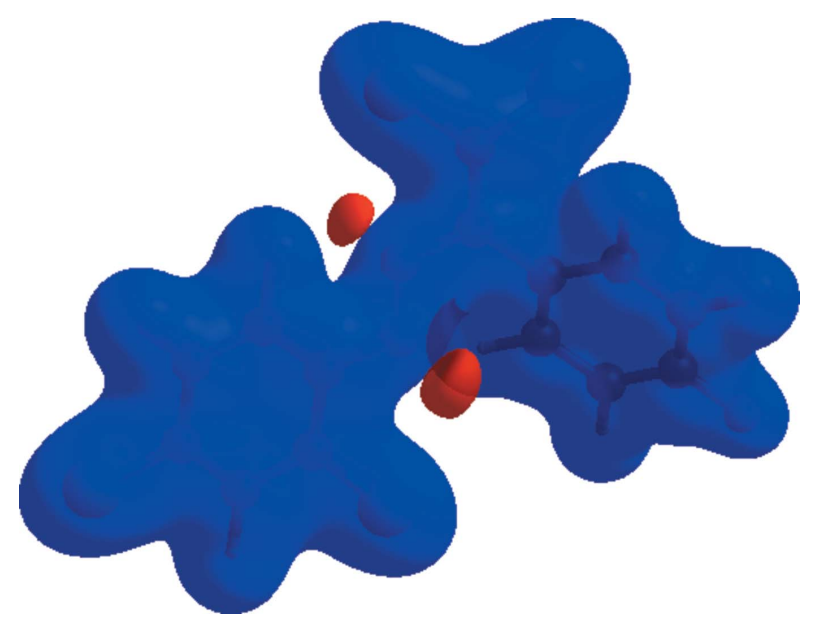

Figure 6

View of the three-dimensional Hirshfeld surface of the title compound plotted over electrostatic potential energy in the range -0.0500 to 0.0500 a.u. using the STO-3 $G$ basis set at the Hartree-Fock level of theory. Hydrogen-bond donors and acceptors are shown as blue and red regions around the atoms, corresponding to positive and negative potentials, respectively. 
Table 3

Percentage contributions of interatomic contacts to the Hirshfeld surface for the title compound..

\begin{tabular}{ll}
\hline Contact & Percentage contribution \\
\hline $\mathrm{Cl} \cdots \mathrm{H} / \mathrm{H} \cdots \mathrm{Cl}$ & 35.1 \\
$\mathrm{H} \cdots \mathrm{H}$ & 10.6 \\
$\mathrm{C} \cdots \mathrm{C}$ & 9.7 \\
$\mathrm{Cl} \cdots \mathrm{Cl}$ & 9.4 \\
$\mathrm{C} \cdots \mathrm{H} / \mathrm{H} \cdots \mathrm{C}$ & 9.2 \\
$\mathrm{Cl} \cdots \mathrm{F} / \mathrm{F} \cdots \mathrm{Cl}$ & 6.7 \\
$\mathrm{C} \cdots \mathrm{C} / \mathrm{C} \cdots \mathrm{Cl}$ & 5.0 \\
$\mathrm{~F} \cdots \mathrm{H} / \mathrm{H} \cdots \mathrm{F}$ & 5.0 \\
$\mathrm{~N} \cdots \mathrm{H} / \mathrm{H} \cdots \mathrm{N}$ & 4.4 \\
$\mathrm{~N} \cdots \mathrm{C} / \mathrm{C} \cdots \mathrm{N}$ & 3.5 \\
$\mathrm{~F} \cdots \mathrm{F}$ & 0.9 \\
$\mathrm{~N} \cdots \mathrm{N}$ & 0.3 \\
$\mathrm{~F} \cdots \mathrm{C} / \mathrm{C} \cdots \mathrm{F}$ & 0.1 \\
\hline
\end{tabular}

The overall two-dimensional fingerprint plot and those delineated into $\mathrm{Cl} \cdots \mathrm{H} / \mathrm{H} \cdots \mathrm{Cl}, \mathrm{H} \cdots \mathrm{H}, \mathrm{C} \cdots \mathrm{C}, \mathrm{Cl} \cdots \mathrm{Cl}$ and $\mathrm{C} \cdots \mathrm{H} / \mathrm{H} \cdots \mathrm{C}$ contacts in the title molecule are illustrated in Fig. 7. The most important interaction is $\mathrm{Cl} \cdots \mathrm{H} / \mathrm{H} \cdots \mathrm{Cl}$, contributing $35.1 \%$ to the overall crystal packing (Fig. $7 b$ ). The secondary important $\mathrm{H} \cdots \mathrm{H}$ and $\mathrm{C} \cdots \mathrm{C}$ interactions contribute $10.6 \%$ (Fig. $7 c$ ) and $9.7 \%$ (Fig. $7 d$ ), respectively, to the Hirshfeld surface. The remaining contributions for the title compound are from $\mathrm{Cl} \cdots \mathrm{Cl}, \mathrm{C} \cdots \mathrm{H} / \mathrm{H} \cdots \mathrm{C}, \mathrm{Cl} \cdots \mathrm{F} / \mathrm{F} \cdots \mathrm{Cl}$, $\mathrm{Cl} \cdots \mathrm{C} / \mathrm{C} \cdots \mathrm{Cl}, \mathrm{F} \cdots \mathrm{H} / \mathrm{H} \cdots \mathrm{F}, \mathrm{N} \cdots \mathrm{H} / \mathrm{H} \cdots \mathrm{N}, \mathrm{N} \cdots \mathrm{N}$ and $\mathrm{F} \cdots \mathrm{C} /$ $\mathrm{C} \cdot \mathrm{F}$ contacts, which are less than $9.7 \%$ and have a negligible effect on the packing. The percentage contributions of all interactions are listed in Table 3.

\section{Database survey}

A search of the Cambridge Structural Database (CSD, Version 5.41, update of November 2019; Groom et al., 2016) for the $(E)$-1-(2,2-dichloro-1-phenylethenyl)-2-phenyldiazene unit resulted in 28 hits. Nine compounds are closely related to the title compound, viz. LEQXOX (I; Shikhaliyev et al., 2018), LEQXIR (II; Shikhaliyev et al., 2018), XIZREG (III; Atioğlu et al., 2019), HODQAV (IV; Shikhaliyev et al., 2019), HONBUK (V; Akkurt et al., 2019), HONBOE (VI; Akkurt et al., 2019), DULTAI (VII; Özkaraca et al., 2020b), GUPHIL (VIII; Özkaraca et al., 2020a) and EBUCUD (IX; Shikhaliyev et al., 2021).

In the crystals of $\mathbf{I}$ and II, the dihedral angles between the aromatic rings are 56.18 (12) and $60.31(14)^{\circ}$, respectively. In I, $\mathrm{C}-\mathrm{H} \cdots \mathrm{N}$ and short $\mathrm{Cl} \cdots \mathrm{Cl}$ contacts are observed and in II, $\mathrm{C}-\mathrm{H} \cdots \mathrm{N}$ and $\mathrm{C}-\mathrm{H} \cdots \mathrm{O}$ hydrogen bonds and short $\mathrm{C}-$ $\mathrm{Cl}$. O contacts occur. In III, the benzene rings form a dihedral angle of $63.29(8)^{\circ}$ and the molecules are linked by $\mathrm{C}-$ $\mathrm{H} \cdot \mathrm{O}$ hydrogen bonds into zigzag chains running along the $c$ axis direction. The crystal packing also features $\mathrm{C}-\mathrm{Cl} \cdots \pi$, $\mathrm{C}-\mathrm{F} \cdots \pi$ and $\mathrm{N}-\mathrm{O} \cdots \pi$ interactions. In $\mathbf{I V}$, the benzene rings make a dihedral angle of $56.13(13)^{\circ}$. Molecules are stacked in columns along the $a$-axis direction via weak $\mathrm{C}-\mathrm{H} \cdots \mathrm{Cl}$ hydrogen bonds and face-to-face $\pi-\pi$ stacking interactions. The crystal packing is further consolidated by short $\mathrm{Cl} \cdots \mathrm{Cl}$ contacts. In $\mathbf{V}$ and VI, the aromatic rings form dihedral angles of 60.9 (2) and $64.1(2)^{\circ}$, respectively. In the crystals, molecules are linked through weak $X \cdots \mathrm{Cl}$ contacts $(X=\mathrm{Cl}$ for $\mathbf{V}$ and $\mathrm{Br}$ for $\mathbf{V I}), \mathrm{C}-\mathrm{H} \cdots \mathrm{Cl}$ and $\mathrm{C}-\mathrm{Cl} \cdots \pi$ interactions into sheets parallel to the $a b$ plane. Additional van der Waals interactions consolidate the three-dimensional packing. In VII, the dihedral angle between the two aromatic rings is $64.12(14)^{\circ}$. The crystal structure is stabilized by a short $\mathrm{C}-\mathrm{H} \cdots \mathrm{Cl}$ contact, $\mathrm{C}-\mathrm{Cl} \cdots \pi$ and van der Waals interactions. In VIII, the benzene rings subtend a dihedral angle of $77.07(10)^{\circ}$. In the crystal, molecules are associated into inversion dimers via short $\mathrm{Cl} \cdots \mathrm{Cl}$ contacts [3.3763 (9) $\AA$ ]. In IX, the asymmetric unit comprises two similar molecules, in which the dihedral angles between the two aromatic rings are 70.1 (3) and $73.2(2)^{\circ}$. The crystal structure features short $\mathrm{C}-\mathrm{H} \cdots \mathrm{Cl}$ and $\mathrm{C}-\mathrm{H} \cdots \mathrm{O}$ contacts and $\mathrm{C}-\mathrm{H} \cdots \pi$ and van der Waals interactions.

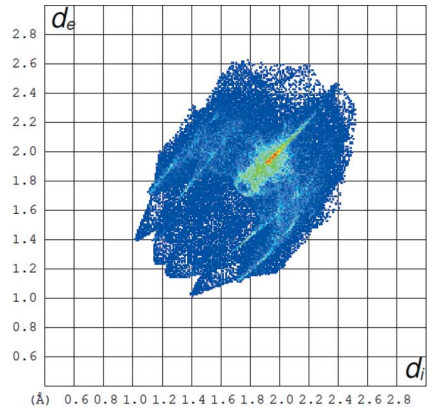

(a) All...All

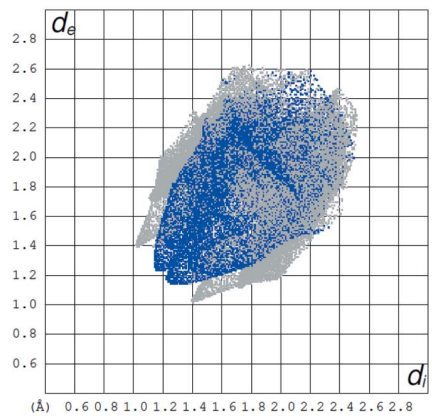

(c) $H \ldots H$

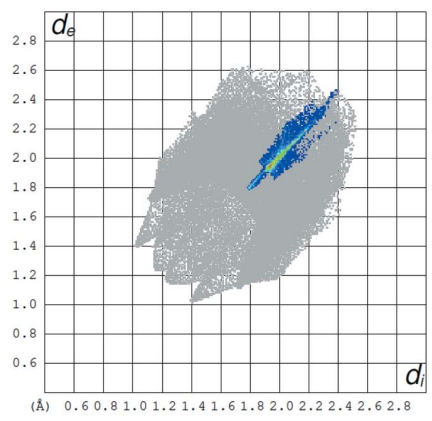

Figure $7 \quad$ (e) $\mathrm{Cl} \cdots \mathrm{Cl}$

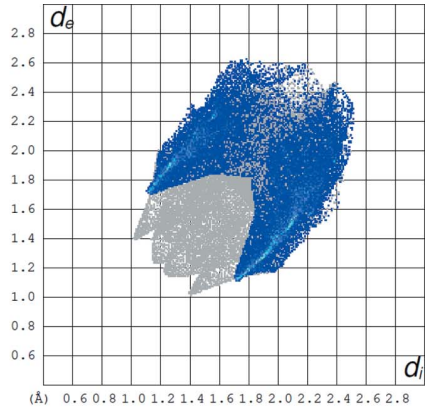

(b) $\mathrm{Cl} \cdots \mathrm{H} / \mathrm{H} \cdots \mathrm{Cl}$

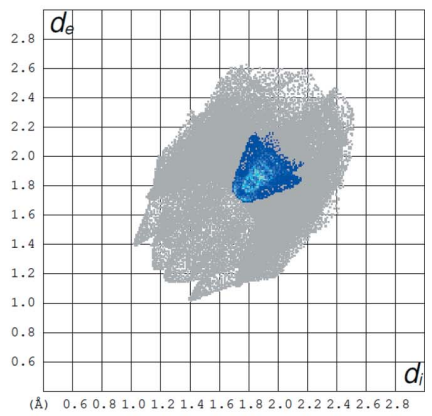

(d) $C \cdots C$

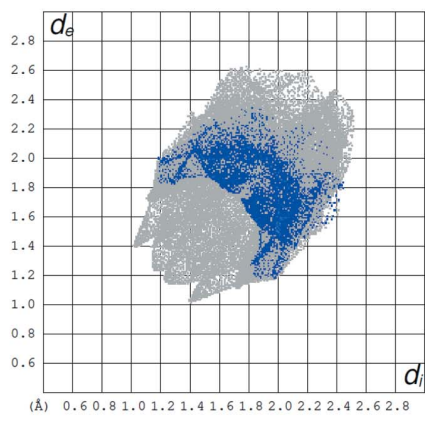

(f) $\mathrm{C} \cdots \boldsymbol{H} / \boldsymbol{H} \cdots \boldsymbol{C}$
The full two-dimensional fingerprint plot for the title compound and those delineated into $(b) \mathrm{Cl} \cdots \mathrm{H} / \mathrm{H} \cdots \mathrm{Cl}(35.1 \%),(c) \mathrm{H} \cdots \mathrm{H}(10.6 \%),(d)$ $\mathrm{C} \cdots \mathrm{C}(9.7 \%),(e) \mathrm{Cl} \cdots \mathrm{Cl}(9.4 \%)$ and $(f) \mathrm{C} \cdots \mathrm{H} / \mathrm{H} \cdots \mathrm{C}(9.2 \%)$ interactions. 


\section{Synthesis and crystallization}

The title dye was synthesized according to the reported method (Shikhaliyev et al., 2018, 2019). A $20 \mathrm{~mL}$ screw-neck vial was charged with DMSO $(10 \mathrm{~mL}),(E)-1-(2,4$-dichlorophenyl)-2-(4-fluorobenzylidene)hydrazine (283 $\mathrm{mg}, 1 \mathrm{mmol})$, tetramethylethylenediamine (TMEDA) (295 mg, $2.5 \mathrm{mmol})$, $\mathrm{CuCl}(2 \mathrm{mg}, 0.02 \mathrm{mmol})$ and $\mathrm{CCl}_{4}$ ( $20 \mathrm{mmol}, 10$ equiv.). After 1-3 h (until TLC analysis showed complete consumption of the corresponding Schiff base), the reaction mixture was poured into $\sim 0.01 M$ solution of $\mathrm{HCl}(100 \mathrm{~mL}, \mathrm{pH}=2-3)$, and extracted with dichloromethane $(3 \times 20 \mathrm{~mL})$. The combined organic phase was washed with water $(3 \times 50 \mathrm{~mL})$ and brine $(30 \mathrm{~mL})$, dried over anhydrous $\mathrm{Na}_{2} \mathrm{SO}_{4}$ and concentrated using a vacuum rotary evaporator. The residue was purified by column chromatography on silica gel using appropriate mixtures of hexane and dichloromethane (3/1-1/1). Crystals suitable for X-ray analysis were obtained by slow evaporation of an ethanol solution. Colourless solid (44\%); m.p. $345 \mathrm{~K}$. Analysis calculated for $\mathrm{C}_{14} \mathrm{H}_{7} \mathrm{Cl}_{4} \mathrm{FN}_{2}(M=364.02)$ : $\mathrm{C} 46.19, \mathrm{H}$ 1.94, N 7.70; found: C 46.11, H 1.98, N 7.67\%. ${ }^{1} \mathrm{H}$ NMR $\left(300 \mathrm{MHz}, \mathrm{CDCl}_{3}\right) \delta 7.31-7.83(7 \mathrm{H}, \mathrm{Ar}) .{ }^{13} \mathrm{C} \mathrm{NMR}(75 \mathrm{MHz}$, $\left.\mathrm{CDCl}_{3}\right) \delta 114.89,115.12,115.41,115.74,115.97,118.33,127.73$, $128.08,128.67,129.17,130.48,132.04,132.15$ and 136.83. ESIMS: $m / z: 365.11[M+\mathrm{H}]^{+}$.

\section{Refinement details}

Crystal data, data collection and structure refinement details are summarized in Table 4. The Moscow synchrotron radiation source was used for the data collection. $\mathrm{H}$ atoms were positioned geometrically and treated as riding atoms where $\mathrm{C}-\mathrm{H}$ $=0.95 \AA$ with $U_{\text {iso }}(\mathrm{H})=1.2 U_{\text {eq }}(\mathrm{C})$. Five outliers $\overline{3} 22, \overline{3} \overline{2} 2$, $\overline{2} 113, \overline{2} 21$ and $\overline{2} \overline{2} 1$ were omitted during the final refinement cycle because of large differences between observed and calculated intensities.

\section{Acknowledgements}

The author's contributions are as follows. Conceptualization, NQS, MA and SM; synthesis, XNB, GTS and MSA; X-ray analysis, KÖ and MA; writing (review and editing of the manuscript), funding acquisition, NQS, XNB, GTS and MSA; supervision, NQS, MA and SM.

\section{Funding information}

This work was performed under the support of the Science Development Foundation under the President of the Republic of Azerbaijan (grant No. EIF-BGM-4- RFTF-1/2017-21/13/4).

\section{References}

Akkurt, M., Shikhaliyev, N. Q., Suleymanova, G. T., Babayeva, G. V., Mammadova, G. Z., Niyazova, A. A., Shikhaliyeva, I. M. \& Toze, F. A. A. (2019). Acta Cryst. E75, 1199-1204.

Atioğlu, Z., Akkurt, M., Shikhaliyev, N. Q., Suleymanova, G. T., Bagirova, K. N. \& Toze, F. A. A. (2019). Acta Cryst. E75, 237-241. Battye, T. G. G., Kontogiannis, L., Johnson, O., Powell, H. R. \& Leslie, A. G. W. (2011). Acta Cryst. D67, 271-281.
Table 4

Experimental details.

\begin{tabular}{ll}
\hline Crystal data & \\
Chemical formula & $\mathrm{C}_{14} \mathrm{H}_{7} \mathrm{Cl}_{4} \mathrm{FN}_{2}$ \\
$M_{\mathrm{r}}$ & 364.02 \\
Crystal system, space group & Monoclinic, $P 2_{1}$ \\
Temperature $(\mathrm{K})$ & 100 \\
$a, b, c(\AA)$ & $3.8720(8), 10.434(2), 18.138(4)$ \\
$\beta\left({ }^{\circ}\right)$ & $95.03(3)$ \\
$V\left(\AA^{3}\right)$ & $730.0(3)$ \\
$Z$ & 2 \\
Radiation type & Synchrotron, $\lambda=0.79475 \AA$ \\
$\mu\left(\mathrm{mm}^{-1}\right)$ & 1.10 \\
Crystal size $(\mathrm{mm})$ & $0.20 \times 0.15 \times 0.10$ \\
& \\
Data collection & Rayonix SX165 CCD \\
Diffractometer & Multi-scan $(S C A L A ;$ Evans, 2006$)$ \\
Absorption correction & $0.800,0.880$ \\
$T_{\min }, T_{\max }$ & $8595,3120,2972$ \\
No. of measured, independent and & \\
$\quad$ observed $[I>2 \sigma(I)]$ reflections & 0.027 \\
$R_{\text {int }}$ & 0.648 \\
$($ sin $\theta / \lambda)$ max $\left(\AA^{-1}\right)$ & \\
& \\
Refinement & $0.036,0.106,1.09$ \\
$R\left[F^{2}>2 \sigma\left(F^{2}\right)\right], w R\left(F^{2}\right), S$ & 3120 \\
No. of reflections & 191 \\
No. of parameters & 1 \\
No. of restraints & $\mathrm{H}$-atom parameters constrained \\
H-atom treatment & $0.61,-0.30$ \\
$\Delta \rho_{\text {max }}, \Delta \rho_{\min }\left(\mathrm{e} \AA^{-3}\right)$ & Flack $x$ determined using 1318 \\
Absolute structure & quotients $\left[\left(I^{+}\right)-\left(I^{-}\right)\right] /\left[\left(I^{+}\right)+\left(I^{-}\right)\right]$ \\
& $($Parsons et al., 2013$)$ \\
Absolute structure parameter & $0.04(2)$ \\
\hline
\end{tabular}

Computer programs: Marccd (Doyle, 2011), iMosflm (Battye et al., 2011), SHELXT (Sheldrick, 2015a), SHELXL (Sheldrick, 2015b), ORTEP-3 for Windows (Farrugia, 2012) and PLATON (Spek, 2020).

Bernstein, J., Davis, R. E., Shimoni, L. \& Chang, N.-L. (1995). Angew. Chem. Int. Ed. Engl. 34, 1555-1573.

Doyle, R. A. (2011). Marccd software manual. Rayonix L. L. C., Evanston, IL 60201, USA.

Evans, P. (2006). Acta Cryst. D62, 72-82.

Farrugia, L. J. (2012). J. Appl. Cryst. 45, 849-854.

Groom, C. R., Bruno, I. J., Lightfoot, M. P. \& Ward, S. C. (2016). Acta Cryst. B72, 171-179.

Gurbanov, A. V., Kuznetsov, M. L., Demukhamedova, S. D., Alieva, I. N., Godjaev, N. M., Zubkov, F. I., Mahmudov, K. T. \& Pombeiro, A. J. L. (2020a). CrystEngComm, 22, 628-633.

Gurbanov, A. V., Kuznetsov, M. L., Mahmudov, K. T., Pombeiro, A. J. L. \& Resnati, G. (2020b). Chem. Eur. J. 26, 14833-14837.

Kopylovich, M. N., Mahmudov, K. T., Mizar, A. \& Pombeiro, A. J. L. (2011). Chem. Commun. 47, 7248-7250.

Ma, Z., Gurbanov, A. V., Maharramov, A. M., Guseinov, F. I., Kopylovich, M. N., Zubkov, F. I., Mahmudov, K. T. \& Pombeiro, A. J. L. (2017a). J. Mol. Catal. A Chem. 426, 526-533.

Ma, Z., Gurbanov, A. V., Sutradhar, M., Kopylovich, M. N., Mahmudov, K. T., Maharramov, A. M., Guseinov, F. I., Zubkov, F. I. \& Pombeiro, A. J. L. (2017b). Mol. Catal. 428, 17-23.

Ma, Z., Mahmudov, K. T., Aliyeva, V. A., Gurbanov, A. V., Guedes da Silva, M. F. C. \& Pombeiro, A. J. L. (2021). Coord. Chem. Rev. 437, 213859.

Ma, Z., Mahmudov, K. T., Aliyeva, V. A., Gurbanov, A. V. \& Pombeiro, A. J. L. (2020). Coord. Chem. Rev. 423, 213482.

Maharramov, A. M., Shikhaliyev, N. Q., Suleymanova, G. T., Gurbanov, A. V., Babayeva, G. V., Mammadova, G. Z., Zubkov, F. I., Nenajdenko, V. G., Mahmudov, K. T. \& Pombeiro, A. J. L. (2018). Dyes Pigments, 159, 135-141. 
Mahmoudi, G., Afkhami, F. A., Castiñeiras, A., García-Santos, I., Gurbanov, A., Zubkov, F. I., Mitoraj, M. P., Kukułka, M., Sagan, F., Szczepanik, D. W., Konyaeva, I. A. \& Safin, D. A. (2018a). Inorg. Chem. 57, 4395-4408.

Mahmoudi, G., Zangrando, E., Mitoraj, M. P., Gurbanov, A. V., Zubkov, F. I., Moosavifar, M., Konyaeva, I. A., Kirillov, A. M. \& Safin, D. A. (2018b). New J. Chem. 42, 4959-4971.

Mahmudov, K. T., Gurbanov, A. V., Aliyeva, V. A., Resnati, G. \& Pombeiro, A. J. L. (2020). Coord. Chem. Rev. 418, 213381.

Mahmudov, K. T., Kopylovich, M. N., Haukka, M., Mahmudova, G. S., Esmaeila, E. F., Chyragov, F. M. \& Pombeiro, A. J. L. (2013). J. Mol. Struct. 1048, 108-112.

Mizar, A., Guedes da Silva, M. F. C., Kopylovich, M. N., Mukherjee, S., Mahmudov, K. T. \& Pombeiro, A. J. L. (2012). Eur. J. Inorg. Chem. pp. 2305-2313.

Özkaraca, K., Akkurt, M., Shikhaliyev, N. Q., Askerova, U. F., Suleymanova, G. T., Mammadova, G. Z. \& Shadrack, D. M. (2020a). Acta Cryst. E76, 1251-1254.

Özkaraca, K., Akkurt, M., Shikhaliyev, N. Q., Askerova, U. F., Suleymanova, G. T., Shikhaliyeva, I. M. \& Bhattarai, A. (2020b). Acta Cryst. E76, 811-815.

Parsons, S., Flack, H. D. \& Wagner, T. (2013). Acta Cryst. B69, 249259.

Sheldrick, G. M. (2015a). Acta Cryst. A71, 3-8.

Sheldrick, G. M. (2015b). Acta Cryst. C71, 3-8.
Shikhaliyev, N. Q., Ahmadova, N. E., Gurbanov, A. V., Maharramov, A. M., Mammadova, G. Z., Nenajdenko, V. G., Zubkov, F. I., Mahmudov, K. T. \& Pombeiro, A. J. L. (2018). Dyes Pigments, 150, 377-381.

Shikhaliyev, N. Q., Atioğlu, Z., Akkurt, M., Qacar, A. M., Askerov, R. K. \& Bhattarai, A. (2021). Acta Cryst. E77, 965-970.

Shikhaliyev, N. Q., Çelikesir, S. T., Akkurt, M., Bagirova, K. N., Suleymanova, G. T. \& Toze, F. A. A. (2019). Acta Cryst. E75, 465469.

Shikhaliyev, N. Q., Kuznetsov, M. L., Maharramov, A. M., Gurbanov, A. V., Ahmadova, N. E., Nenajdenko, V. G., Mahmudov, K. T. \& Pombeiro, A. J. L. (2019). CrystEngComm, 21, 5032-5038.

Shixaliyev, N. Q., Gurbanov, A. V., Maharramov, A. M., Mahmudov, K. T., Kopylovich, M. N., Martins, L. M. D. R. S., Muzalevskiy, V. M., Nenajdenko, V. G. \& Pombeiro, A. J. L. (2014). New J. Chem. 38, 4807-4815.

Spackman, M. A., McKinnon, J. J. \& Jayatilaka, D. (2008). CrystEngComm, 10, 377-388.

Spek, A. L. (2020). Acta Cryst. E76, 1-11.

Turner, M. J., McKinnon, J. J., Wolff, S. K., Grimwood, D. J., Spackman, P. R., Jayatilaka, D. \& Spackman, M. A. (2017). CrystalExplorer17. The University of Western Australia.

Viswanathan, A., Kute, D., Musa, A., Konda Mani, S., Sipilä, V., Emmert-Streib, F., Zubkov, F. I., Gurbanov, A. V., Yli-Harja, O. \& Kandhavelu, M. (2019). Eur. J. Med. Chem. 166, 291-303. 


\section{supporting information}

Acta Cryst. (2021). E77, 1158-1163 [https://doi.org/10.1107/S2056989021010756]

Crystal structure and Hirshfeld surface analysis of (E)-1-[2,2-dichloro-1-(4fluorophenyl)ethenyl]-2-(2,4-dichlorophenyl)diazene

Namiq Q. Shikhaliyev, Kadiriye Özkaraca, Mehmet Akkurt, Xanim N. Bagirova, Gulnar T.

Suleymanova, Mirjalil S. Abdulov and Sixberth Mlowe

Computing details

Data collection: Marccd (Doyle, 2011); cell refinement: iMosflm (Battye et al., 2011); data reduction: iMosflm (Battye et al., 2011); program(s) used to solve structure: SHELXT (Sheldrick, 2015a); program(s) used to refine structure: SHELXL (Sheldrick, 2015b); molecular graphics: ORTEP-3 for Windows (Farrugia, 2012); software used to prepare material for publication: PLATON (Spek, 2020).

(E)-1-[2,2-Dichloro-1-(4-fluorophenyl)ethenyl]-2-(2,4-dichlorophenyl)diazene

Crystal data

$\mathrm{C}_{14} \mathrm{H}_{7} \mathrm{Cl}_{4} \mathrm{FN}_{2}$

$M_{r}=364.02$

Monoclinic, $P 2_{1}$

$a=3.8720(8) \AA$

$b=10.434(2) \AA$

$c=18.138(4) \AA$

$\beta=95.03(3)^{\circ}$

$V=730.0(3) \AA^{3}$

$Z=2$

\section{Data collection}

Rayonix SX165 CCD

diffractometer

/f scan

Absorption correction: multi-scan

(Scala; Evans, 2006)

$T_{\min }=0.800, T_{\max }=0.880$

8595 measured reflections

\section{Refinement}

Refinement on $F^{2}$

Least-squares matrix: full

$R\left[F^{2}>2 \sigma\left(F^{2}\right)\right]=0.036$

$w R\left(F^{2}\right)=0.106$

$S=1.09$

3120 reflections

191 parameters

1 restraint
$F(000)=364$

$D_{\mathrm{x}}=1.656 \mathrm{Mg} \mathrm{m}^{-3}$

Synchrotron radiation, $\lambda=0.79475 \AA$

Cell parameters from 600 reflections

$\theta=2.8-28.0^{\circ}$

$\mu=1.12 \mathrm{~mm}^{-1}$

$T=100 \mathrm{~K}$

Prism, colourless

$0.20 \times 0.15 \times 0.10 \mathrm{~mm}$

3120 independent reflections

2972 reflections with $I>2 \sigma(I)$

$R_{\text {int }}=0.027$

$\theta_{\text {max }}=31.0^{\circ}, \theta_{\text {min }}=2.5^{\circ}$

$h=-5 \rightarrow 5$

$k=-12 \rightarrow 13$

$l=-23 \rightarrow 23$

Hydrogen site location: inferred from neighbouring sites

$\mathrm{H}$-atom parameters constrained

$w=1 /\left[\sigma^{2}\left(F_{\mathrm{o}}{ }^{2}\right)+(0.0549 P)^{2}+0.7552 P\right]$

where $P=\left(F_{\mathrm{o}}^{2}+2 F_{\mathrm{c}}^{2}\right) / 3$

$(\Delta / \sigma)_{\max }<0.001$

$\Delta \rho_{\max }=0.61 \mathrm{e} \AA^{-3}$

$\Delta \rho_{\min }=-0.30$ e $\AA^{-3}$ 
Extinction correction: SHELXL,

$\mathrm{Fc}^{*}=\mathrm{kFc}\left[1+0.001 \times \mathrm{xc}^{2} \lambda^{3} / \sin (2 \theta)\right]^{-1 / 4}$

Extinction coefficient: $0.044(8)$
Absolute structure: Flack $x$ determined using

1318 quotients $\left[\left(I^{+}\right)-\left(I^{-}\right)\right] /\left[\left(I^{+}\right)+\left(I^{-}\right)\right]$(Parsons et al., 2013)

Absolute structure parameter: 0.04 (3)

\section{Special details}

Geometry. All esds (except the esd in the dihedral angle between two 1.s. planes) are estimated using the full covariance matrix. The cell esds are taken into account individually in the estimation of esds in distances, angles and torsion angles; correlations between esds in cell parameters are only used when they are defined by crystal symmetry. An approximate (isotropic) treatment of cell esds is used for estimating esds involving l.s. planes.

Fractional atomic coordinates and isotropic or equivalent isotropic displacement parameters $\left(\AA^{2}\right)$

\begin{tabular}{lllll}
\hline & $x$ & $y$ & $z$ & $U_{\text {iso }} / U_{\text {eq }}$ \\
\hline C11 & $-0.3214(3)$ & $0.89506(12)$ & $0.70389(6)$ & $0.0309(3)$ \\
C12 & $-0.0221(4)$ & $0.81658(13)$ & $0.84672(7)$ & $0.0392(3)$ \\
C13 & $0.2392(3)$ & $0.19949(12)$ & $0.70702(6)$ & $0.0334(3)$ \\
C14 & $0.8795(3)$ & $0.10530(13)$ & $0.97679(6)$ & $0.0340(3)$ \\
F1 & $-0.2885(9)$ & $0.5192(3)$ & $0.42861(16)$ & $0.0383(7)$ \\
N1 & $0.1181(11)$ & $0.5745(4)$ & $0.7820(2)$ & $0.0281(9)$ \\
N2 & $0.1962(11)$ & $0.4658(4)$ & $0.7569(2)$ & $0.0273(8)$ \\
C1 & $-0.0396(12)$ & $0.6583(5)$ & $0.7275(3)$ & $0.0274(9)$ \\
C2 & $-0.1180(12)$ & $0.7756(5)$ & $0.7555(3)$ & $0.0293(10)$ \\
C3 & $-0.1089(12)$ & $0.6232(5)$ & $0.6480(2)$ & $0.0255(9)$ \\
C4 & $-0.2763(12)$ & $0.5073(5)$ & $0.6280(3)$ & $0.0267(9)$ \\
H4 & -0.347529 & 0.452035 & 0.665430 & $0.032^{*}$ \\
C5 & $-0.3391(13)$ & $0.4726(5)$ & $0.5540(3)$ & $0.0288(10)$ \\
H5 & -0.455606 & 0.394787 & 0.540530 & $0.035^{*}$ \\
C6 & $-0.2292(13)$ & $0.5531(5)$ & $0.5008(3)$ & $0.0295(10)$ \\
C7 & $-0.0634(12)$ & $0.6678(5)$ & $0.5181(3)$ & $0.0289(10)$ \\
H7 & 0.006934 & 0.722093 & 0.480114 & $0.035^{*}$ \\
C8 & $-0.0016(12)$ & $0.7022(5)$ & $0.5920(3)$ & $0.0281(9)$ \\
H8 & 0.114818 & 0.780287 & 0.604727 & $0.034^{*}$ \\
C9 & $0.3594(11)$ & $0.3839(5)$ & $0.8125(2)$ & $0.0261(9)$ \\
C10 & $0.3960(12)$ & $0.2556(5)$ & $0.7935(3)$ & $0.0265(9)$ \\
C11 & $0.5577(13)$ & $0.1679(5)$ & $0.8440(3)$ & $0.0275(9)$ \\
H11 & 0.581566 & 0.080365 & 0.830976 & $0.033^{*}$ \\
C12 & $0.6813(13)$ & $0.2120(5)$ & $0.9131(3)$ & $0.0291(10)$ \\
C13 & $0.6495(12)$ & $0.3405(5)$ & $0.9334(3)$ & $0.0289(10)$ \\
H13 & 0.736480 & 0.368972 & 0.981195 & $0.035^{*}$ \\
C14 & $0.4893(13)$ & $0.4255(5)$ & $0.8827(3)$ & $0.0300(10)$ \\
H14 & 0.467401 & 0.513039 & 0.895888 & $0.036^{*}$ \\
& & & &
\end{tabular}

Atomic displacement parameters $\left(\AA^{2}\right)$

\begin{tabular}{lllllll}
\hline & $U^{11}$ & $U^{22}$ & $U^{33}$ & $U^{12}$ & $U^{13}$ & $U^{23}$ \\
\hline C11 & $0.0349(6)$ & $0.0218(6)$ & $0.0353(6)$ & $0.0044(5)$ & $-0.0005(4)$ & $-0.0001(4)$ \\
C12 & $0.0528(8)$ & $0.0325(7)$ & $0.0312(6)$ & $0.0116(6)$ & $-0.0029(5)$ & $-0.0065(5)$ \\
C13 & $0.0426(6)$ & $0.0263(6)$ & $0.0301(6)$ & $0.0037(5)$ & $-0.0030(4)$ & $-0.0036(4)$
\end{tabular}


supporting information

$\begin{array}{lllllll}\text { C14 } & 0.0385(6) & 0.0315(7) & 0.0318(5) & 0.0058(5) & 0.0018(4) & 0.0071(5) \\ \text { F1 } & 0.0507(18) & 0.0339(18) & 0.0296(15) & 0.0000(14) & 0.0002(12) & -0.0029(12) \\ \text { N1 } & 0.031(2) & 0.022(2) & 0.032(2) & 0.0034(16) & 0.0042(15) & -0.0011(15) \\ \text { N2 } & 0.028(2) & 0.025(2) & 0.0291(19) & 0.0041(15) & 0.0052(15) & 0.0005(15) \\ \text { C1 } & 0.027(2) & 0.023(2) & 0.033(2) & 0.0038(17) & 0.0032(17) & 0.0004(18) \\ \text { C2 } & 0.027(2) & 0.027(3) & 0.034(2) & 0.0060(18) & 0.0015(18) & -0.0031(19) \\ \text { C3 } & 0.027(2) & 0.020(2) & 0.029(2) & 0.0037(17) & 0.0026(16) & 0.0005(17) \\ \text { C4 } & 0.028(2) & 0.019(2) & 0.034(2) & 0.0029(17) & 0.0062(17) & 0.0004(17) \\ \text { C5 } & 0.029(2) & 0.022(2) & 0.036(2) & 0.0031(17) & 0.0024(18) & 0.0000(18) \\ \text { C6 } & 0.030(2) & 0.030(3) & 0.028(2) & 0.0053(18) & 0.0009(17) & -0.0018(17) \\ \text { C7 } & 0.028(2) & 0.025(3) & 0.033(2) & 0.0024(17) & 0.0034(17) & 0.0035(18) \\ \text { C8 } & 0.028(2) & 0.023(2) & 0.033(2) & 0.0029(19) & 0.0019(16) & 0.0018(19) \\ \text { C9 } & 0.024(2) & 0.026(2) & 0.029(2) & 0.0012(18) & 0.0052(15) & 0.0031(18) \\ \text { C10 } & 0.029(2) & 0.024(2) & 0.027(2) & 0.0040(18) & 0.0044(17) & -0.0001(17) \\ \text { C11 } & 0.029(2) & 0.024(3) & 0.030(2) & 0.0045(17) & 0.0046(16) & 0.0013(17) \\ \text { C12 } & 0.029(2) & 0.027(3) & 0.032(2) & 0.0030(19) & 0.0055(17) & 0.0057(19) \\ \text { C13 } & 0.031(2) & 0.029(3) & 0.026(2) & -0.0010(19) & 0.0017(17) & -0.0041(18) \\ \text { C14 } & 0.035(2) & 0.024(3) & 0.031(2) & 0.0021(18) & 0.0043(18) & 0.0002(18) \\ & & & & & & \end{array}$

Geometric parameters $\left(\AA,{ }^{o}\right)$

\begin{tabular}{|c|c|c|c|}
\hline $\mathrm{C} 11-\mathrm{C} 2$ & $1.709(5)$ & $\mathrm{C} 5-\mathrm{H} 5$ & 0.9500 \\
\hline $\mathrm{Cl} 2-\mathrm{C} 2$ & $1.718(5)$ & $\mathrm{C} 6-\mathrm{C} 7$ & $1.381(7)$ \\
\hline $\mathrm{Cl} 3-\mathrm{C} 10$ & $1.733(5)$ & $\mathrm{C} 7-\mathrm{C} 8$ & $1.388(7)$ \\
\hline $\mathrm{Cl} 4-\mathrm{C} 12$ & $1.735(5)$ & $\mathrm{C} 7-\mathrm{H} 7$ & 0.9500 \\
\hline $\mathrm{F} 1-\mathrm{C} 6$ & $1.357(6)$ & $\mathrm{C} 8-\mathrm{H} 8$ & 0.9500 \\
\hline $\mathrm{N} 1-\mathrm{N} 2$ & $1.269(6)$ & $\mathrm{C} 9-\mathrm{C} 10$ & $1.393(7)$ \\
\hline $\mathrm{N} 1-\mathrm{C} 1$ & $1.416(6)$ & C9- & $1.398(7)$ \\
\hline $\mathrm{N} 2-\mathrm{C} 9$ & $1.426(6)$ & $\mathrm{C} 10-\mathrm{C} 11$ & $1.403(7)$ \\
\hline $\mathrm{C} 1-\mathrm{C} 2$ & $1.369(7)$ & $\mathrm{C} 11-\mathrm{C} 12$ & $1.380(7)$ \\
\hline $\mathrm{C} 1-\mathrm{C} 3$ & $1.490(6)$ & C11-H11 & 0.9500 \\
\hline $\mathrm{C} 3-\mathrm{C} 8$ & $1.399(7)$ & $\mathrm{C} 12-\mathrm{C} 13$ & $1.399(7)$ \\
\hline $\mathrm{C} 3-\mathrm{C} 4$ & $1.404(7)$ & $\mathrm{C} 13-\mathrm{C} 14$ & $1.384(7)$ \\
\hline $\mathrm{C} 4-\mathrm{C} 5$ & $1.390(7)$ & $\mathrm{C} 13-\mathrm{H} 13$ & 0.9500 \\
\hline $\mathrm{C} 4-\mathrm{H} 4$ & 0.9500 & $\mathrm{C} 14-\mathrm{H} 14$ & 0.9500 \\
\hline $\mathrm{C} 5-\mathrm{C} 6$ & $1.375(7)$ & & \\
\hline $\mathrm{N} 2-\mathrm{N} 1-\mathrm{C} 1$ & $113.8(4)$ & $\mathrm{C} 8-\mathrm{C} 7-\mathrm{H} 7$ & 120.7 \\
\hline $\mathrm{N} 1-\mathrm{N} 2-\mathrm{C} 9$ & $112.8(4)$ & $\mathrm{C} 7-\mathrm{C} 8-\mathrm{C} 3$ & $120.8(5)$ \\
\hline $\mathrm{C} 2-\mathrm{C} 1-\mathrm{N} 1$ & $112.9(4)$ & $\mathrm{C} 7-\mathrm{C} 8-\mathrm{H} 8$ & 119.6 \\
\hline $\mathrm{C} 2-\mathrm{C} 1-\mathrm{C} 3$ & $123.4(4)$ & $\mathrm{C} 3-\mathrm{C} 8-\mathrm{H} 8$ & 119.6 \\
\hline $\mathrm{N} 1-\mathrm{C} 1-\mathrm{C} 3$ & $123.6(4)$ & $\mathrm{C} 10-\mathrm{C} 9-\mathrm{C} 14$ & $119.2(4)$ \\
\hline $\mathrm{C} 1-\mathrm{C} 2-\mathrm{C} 11$ & $123.8(4)$ & $\mathrm{C} 10-\mathrm{C} 9-\mathrm{N} 2$ & $116.7(4)$ \\
\hline $\mathrm{C} 1-\mathrm{C} 2-\mathrm{Cl} 2$ & $122.9(4)$ & $\mathrm{C} 14-\mathrm{C} 9-\mathrm{N} 2$ & $124.1(5)$ \\
\hline $\mathrm{C} 11-\mathrm{C} 2-\mathrm{Cl} 2$ & $113.3(3)$ & $\mathrm{C} 9-\mathrm{C} 10-\mathrm{C} 11$ & $121.0(4)$ \\
\hline $\mathrm{C} 8-\mathrm{C} 3-\mathrm{C} 4$ & $118.7(4)$ & $\mathrm{C} 9-\mathrm{C} 10-\mathrm{Cl} 3$ & $120.9(4)$ \\
\hline $\mathrm{C} 8-\mathrm{C} 3-\mathrm{C} 1$ & $121.2(4)$ & $\mathrm{C} 11-\mathrm{C} 10-\mathrm{Cl} 3$ & $118.1(4)$ \\
\hline $\mathrm{C} 4-\mathrm{C} 3-\mathrm{C} 1$ & $120.1(4)$ & $\mathrm{C} 12-\mathrm{C} 11-\mathrm{C} 10$ & $118.4(5)$ \\
\hline
\end{tabular}




\begin{tabular}{|c|c|c|c|}
\hline $\mathrm{C} 5-\mathrm{C} 4-\mathrm{C} 3$ & $120.8(4)$ & $\mathrm{C} 12-\mathrm{C} 11-\mathrm{H} 11$ & 120.8 \\
\hline $\mathrm{C} 5-\mathrm{C} 4-\mathrm{H} 4$ & 119.6 & $\mathrm{C} 10-\mathrm{C} 11-\mathrm{H} 11$ & 120.8 \\
\hline $\mathrm{C} 3-\mathrm{C} 4-\mathrm{H} 4$ & 119.6 & $\mathrm{C} 11-\mathrm{C} 12-\mathrm{C} 13$ & $121.8(5)$ \\
\hline $\mathrm{C} 6-\mathrm{C} 5-\mathrm{C} 4$ & $118.6(5)$ & $\mathrm{C} 11-\mathrm{C} 12-\mathrm{Cl} 4$ & $119.3(4)$ \\
\hline $\mathrm{C} 6-\mathrm{C} 5-\mathrm{H} 5$ & 120.7 & $\mathrm{C} 13-\mathrm{C} 12-\mathrm{Cl} 4$ & $118.9(4)$ \\
\hline $\mathrm{C} 4-\mathrm{C} 5-\mathrm{H} 5$ & 120.7 & $\mathrm{C} 14-\mathrm{C} 13-\mathrm{C} 12$ & $118.9(4)$ \\
\hline $\mathrm{F} 1-\mathrm{C} 6-\mathrm{C} 5$ & $118.8(5)$ & $\mathrm{C} 14-\mathrm{C} 13-\mathrm{H} 13$ & 120.5 \\
\hline $\mathrm{F} 1-\mathrm{C} 6-\mathrm{C} 7$ & $118.7(5)$ & $\mathrm{C} 12-\mathrm{C} 13-\mathrm{H} 13$ & 120.5 \\
\hline $\mathrm{C} 5-\mathrm{C} 6-\mathrm{C} 7$ & $122.5(5)$ & $\mathrm{C} 13-\mathrm{C} 14-\mathrm{C} 9$ & $120.7(5)$ \\
\hline $\mathrm{C} 6-\mathrm{C} 7-\mathrm{C} 8$ & $118.7(5)$ & $\mathrm{C} 13-\mathrm{C} 14-\mathrm{H} 14$ & 119.6 \\
\hline $\mathrm{C} 6-\mathrm{C} 7-\mathrm{H} 7$ & 120.7 & C9-C14-H14 & 119.6 \\
\hline $\mathrm{C} 1-\mathrm{N} 1-\mathrm{N} 2-\mathrm{C} 9$ & $179.1(4)$ & $\mathrm{C} 6-\mathrm{C} 7-\mathrm{C} 8-\mathrm{C} 3$ & $-0.8(7)$ \\
\hline $\mathrm{N} 2-\mathrm{N} 1-\mathrm{C} 1-\mathrm{C} 2$ & $-179.2(4)$ & $\mathrm{C} 4-\mathrm{C} 3-\mathrm{C} 8-\mathrm{C} 7$ & $0.9(7)$ \\
\hline $\mathrm{N} 2-\mathrm{N} 1-\mathrm{C} 1-\mathrm{C} 3$ & $0.0(7)$ & $\mathrm{C} 1-\mathrm{C} 3-\mathrm{C} 8-\mathrm{C} 7$ & $179.1(4)$ \\
\hline $\mathrm{N} 1-\mathrm{C} 1-\mathrm{C} 2-\mathrm{Cl} 1$ & $-178.0(4)$ & $\mathrm{N} 1-\mathrm{N} 2-\mathrm{C} 9-\mathrm{C} 10$ & $168.4(4)$ \\
\hline $\mathrm{C} 3-\mathrm{C} 1-\mathrm{C} 2-\mathrm{C} 11$ & $2.8(7)$ & $\mathrm{N} 1-\mathrm{N} 2-\mathrm{C} 9-\mathrm{C} 14$ & $-13.2(7)$ \\
\hline $\mathrm{N} 1-\mathrm{C} 1-\mathrm{C} 2-\mathrm{Cl} 2$ & $1.7(6)$ & $\mathrm{C} 14-\mathrm{C} 9-\mathrm{C} 10-\mathrm{C} 11$ & $0.6(7)$ \\
\hline $\mathrm{C} 3-\mathrm{C} 1-\mathrm{C} 2-\mathrm{Cl} 2$ & $-177.6(4)$ & $\mathrm{N} 2-\mathrm{C} 9-\mathrm{C} 10-\mathrm{C} 11$ & $179.1(4)$ \\
\hline $\mathrm{C} 2-\mathrm{C} 1-\mathrm{C} 3-\mathrm{C} 8$ & $50.4(7)$ & $\mathrm{C} 14-\mathrm{C} 9-\mathrm{C} 10-\mathrm{Cl} 3$ & $180.0(4)$ \\
\hline $\mathrm{N} 1-\mathrm{C} 1-\mathrm{C} 3-\mathrm{C} 8$ & $-128.7(5)$ & $\mathrm{N} 2-\mathrm{C} 9-\mathrm{C} 10-\mathrm{Cl} 3$ & $-1.6(6)$ \\
\hline $\mathrm{C} 2-\mathrm{C} 1-\mathrm{C} 3-\mathrm{C} 4$ & $-131.3(5)$ & $\mathrm{C} 9-\mathrm{C} 10-\mathrm{C} 11-\mathrm{C} 12$ & $-0.2(7)$ \\
\hline $\mathrm{N} 1-\mathrm{C} 1-\mathrm{C} 3-\mathrm{C} 4$ & $49.5(6)$ & $\mathrm{Cl} 3-\mathrm{C} 10-\mathrm{C} 11-\mathrm{C} 12$ & $-179.6(4)$ \\
\hline $\mathrm{C} 8-\mathrm{C} 3-\mathrm{C} 4-\mathrm{C} 5$ & $-0.9(7)$ & $\mathrm{C} 10-\mathrm{C} 11-\mathrm{C} 12-\mathrm{C} 13$ & $-0.1(7)$ \\
\hline $\mathrm{C} 1-\mathrm{C} 3-\mathrm{C} 4-\mathrm{C} 5$ & $-179.2(4)$ & $\mathrm{C} 10-\mathrm{C} 11-\mathrm{C} 12-\mathrm{Cl} 4$ & $179.5(4)$ \\
\hline $\mathrm{C} 3-\mathrm{C} 4-\mathrm{C} 5-\mathrm{C} 6$ & $1.0(7)$ & $\mathrm{C} 11-\mathrm{C} 12-\mathrm{C} 13-\mathrm{C} 14$ & $0.1(7)$ \\
\hline $\mathrm{C} 4-\mathrm{C} 5-\mathrm{C} 6-\mathrm{F} 1$ & $179.7(4)$ & $\mathrm{C} 14-\mathrm{C} 12-\mathrm{C} 13-\mathrm{C} 14$ & $-179.6(4)$ \\
\hline $\mathrm{C} 4-\mathrm{C} 5-\mathrm{C} 6-\mathrm{C} 7$ & $-0.9(7)$ & $\mathrm{C} 12-\mathrm{C} 13-\mathrm{C} 14-\mathrm{C} 9$ & $0.3(7)$ \\
\hline $\mathrm{F} 1-\mathrm{C} 6-\mathrm{C} 7-\mathrm{C} 8$ & $-179.8(4)$ & $\mathrm{C} 10-\mathrm{C} 9-\mathrm{C} 14-\mathrm{C} 13$ & $-0.7(7)$ \\
\hline $\mathrm{C} 5-\mathrm{C} 6-\mathrm{C} 7-\mathrm{C} 8$ & $0.9(7)$ & $\mathrm{N} 2-\mathrm{C} 9-\mathrm{C} 14-\mathrm{C} 13$ & $-179.0(4)$ \\
\hline
\end{tabular}

Hydrogen-bond geometry $\left(A,{ }^{\circ}\right)$

$\mathrm{Cg} 2$ is the centroid of the C9-C14 2,4-dichlorophenyl ring.

\begin{tabular}{lllll}
\hline$D-\mathrm{H} \cdots A$ & $D-\mathrm{H}$ & $\mathrm{H} \cdots A$ & $D \cdots A$ & $D-\mathrm{H} \cdots A$ \\
\hline $\mathrm{C} 4-\mathrm{H} 4 \cdots \mathrm{N} 2^{\mathrm{i}}$ & 0.95 & 2.53 & $3.265(6)$ & 134 \\
$\mathrm{C} 12-\mathrm{C} 14 \cdots C g 2^{\mathrm{ii}}$ & $1.74(1)$ & $3.92(1)$ & $3.569(6)$ & $66(1)$ \\
\hline
\end{tabular}

Symmetry codes: (i) $x-1, y, z$; (ii) $x+1, y, z$. 